Draft Version June 16, 2020

Typeset using $\mathrm{LAT}_{\mathrm{E}} \mathrm{X}$ twocolumn style in AASTeX63

\title{
The Birth of a Massive First-Star Binary
}

\author{
Kazuyuki Sugimura, ${ }^{1,2}$ Tomoaki Matsumoto, ${ }^{3}$ Takashi Hosokawa, ${ }^{4}$ Shingo Hirano, ${ }^{5}$ And Kazuyuki Omukai ${ }^{2}$ \\ ${ }^{1}$ Department of Astronomy, University of Maryland, College Park, MD 20740, USA \\ ${ }^{2}$ Astronomical Institute, Graduate School of Science, Tohoku University, Aoba, Sendai 980-8578, Japan \\ ${ }^{3}$ Faculty of Sustainability Studies, Hosei University, Fujimi, Chiyoda, Tokyo 102-8160, Japan \\ ${ }^{4}$ Department of Physics, Kyoto University, Sakyo, Kyoto 606-8502, Japan \\ ${ }^{5}$ Faculty of Sciences, Department of Earth and Planetary Sciences, Kyushu University, Fukuoka 812-8581, Japan
}

\begin{abstract}
We study the formation of massive Population III binary stars using a newly developed radiation hydrodynamics code with the adaptive mesh refinement and adaptive ray-tracing methods. We follow the evolution of a typical primordial star-forming cloud obtained from a cosmological hydrodynamics simulation. Several protostars form as a result of disk fragmentation and grow in mass by the gas accretion, which is finally quenched by the radiation feedback from the protostars. Our code enables us, for the first time, to consider the feedback by both the ionizing and dissociating radiation from the multiple protostars, which is essential for self-consistently determining their final masses. At the final step of the simulation, we observe a very wide $\left(\gtrsim 10^{4} \mathrm{au}\right)$ binary stellar system consisting of 60 and $70 M_{\odot}$ stars. One of the member stars also has two smaller mass $\left(10 M_{\odot}\right)$ companion stars orbiting at 200 and $800 \mathrm{au}$, making up a mini-triplet system. Our results suggest that massive binary or multiple systems are common among Population III stars.
\end{abstract}

Keywords: cosmology: theory — early universe — stars: formation — stars: population III

\section{INTRODUCTION}

The first generation of stars in the Universe, also known as the Population III (Pop III) stars, are born in minihalos of $10^{5}-10^{6} M_{\odot}$ around the redshift $z \sim$ $20-30$ in the framework of the standard Lambda cold dark matter $(\Lambda \mathrm{CDM})$ cosmology (e.g., Abel et al. 2002; Bromm et al. 2002; see also Glover 2013; Greif 2015 for review). Small embryonic protostars formed by the gravitational collapse of the natal clouds (e.g., Omukai \& Nishi 1998; Yoshida et al. 2008) grow in mass by accretion of the surrounding gas (e.g., Omukai \& Palla 2003; Tan \& McKee 2004), finally reaching the mass of 10-1000 $M_{\odot}$ (e.g., Susa et al. 2014; Hirano et al. 2015; Hosokawa et al. 2016, hereafter H16) when the radiation feedback quenches the gas supply (e.g., Omukai \& Inutsuka 2002; McKee \& Tan 2008; Hosokawa et al. 2011). If born as binaries, such massive stars can be the progenitors of the binary black holes (BHs) with masses $\gtrsim 30 M_{\odot}$ recently observed in gravitational waves (e.g., Kinugawa et al. 2014; Hartwig et al. 2016; Abbott et al.

sugimura@astro.umd.edu
2016, but also see Belczynski et al. 2017), as well as the X-ray binaries that affect the thermal history of the intergalactic medium (Mirabel et al. 2011; Jeon et al. 2014 ), which is a target of future $21 \mathrm{~cm}$ line observations (e.g., Dewdney et al. 2009).

Recent numerical simulations have shown that the disks around Pop III protostars are gravitationally unstable and fragment into clumps, in which other protostars can form (e.g., Stacy et al. 2010, 2012, 2016; Clark et al. 2011; Greif et al. 2011, 2012; Smith et al. 2011; Hirano \& Bromm 2017; Sharda et al. 2019). Although most of these protostars merge with another protostar soon after their formation, some of them may survive for a longer period and may end up with binary or multiple systems (e.g., Susa 2019; Chon \& Hosokawa 2019). Unfortunately, however, those simulations covered only early phases of the star formation process, and thus the fate of the protostars remains unknown. To reveal the nature of resulting stellar systems, we need to follow not only the formation of multiple protostars by disk fragmentation but also their long-term evolution under the influence of the protostellar radiation.

In this work, we perform simulation of Pop III star formation, self-consistently taking into account the ra- 
diation from multiple protostars, by using a newly developed radiation hydrodynamics code, SFUMATO-RT. We follow the entire star formation process until the protostellar radiation feedback terminates the gas accretion to the newborn stellar system. In this Letter, we describe results of a run with a typical initial condition for the primordial star formation. Results for various cases with further analysis, together with a detailed description of our code, will be presented in a forthcoming publication (Sugimura et al., in preparation).

\section{NUMERICAL METHOD}

\subsection{Radiation hydrodynamics simulation}

We use a new radiation hydrodynamics code, SFUMATO-RT, which is a modified version of a selfgravitational magnetohydrodynamics code with adaptive mesh refinement (AMR), SFUMATO (Matsumoto 2007; Matsumoto et al. 2015). We have newly added a chemistry module coupled with radiation transfer (RT) to consider the thermal evolution of the primordial gas under the influence of the radiation from protostars. The hydrodynamical scheme has second-order accuracy in space and time.

The model of primordial chemistry and thermodynamics is basically the same as in H16. We solve the nonequilibrium chemical reactions among six species, $\mathrm{H}^{+}$, $\mathrm{H}, \mathrm{H}_{2}, \mathrm{H}^{-}, \mathrm{H}_{2}^{+}$, and $\mathrm{e}^{-}$, assuming all He to be neutral. We consider chemical reactions and heating/cooling processes relevant in the density range $n_{\mathrm{H}}<10^{13} \mathrm{~cm}^{-3}$, where $n_{\mathrm{H}}$ is the number density of hydrogen nuclei.

Protostars are represented by sink particles, which interact with the gas through gravity and accretion. The threshold density for particle creation is set at $n_{\text {sink }}=2 \times 10^{11} \mathrm{~cm}^{-3}$. The particles accrete the gas within the sink radius $r_{\text {sink }}=64 \mathrm{au}$, which is equal to a half the Jeans length for molecular gas with $n_{\mathrm{H}}=n_{\text {sink }}$ and $T=1000 \mathrm{~K}$. Particles are assumed to merge when their distance becomes shorter than $2 r_{\text {sink }}$.

We calculate the radiative property of Pop III protostars using a pre-calculated table obtained with a onedimensional stellar evolution code under the assumption of constant accretion rates (Hosokawa \& Omukai 2009; Hosokawa et al. 2010). The table gives the emission rates of extreme-ultraviolet (EUV; $h \nu>13.6 \mathrm{eV}$ ) ionizing and far-ultraviolet (FUV; $11.2 \mathrm{eV}<h \nu<13.6 \mathrm{eV}$ ) dissociating photons for a given set of the stellar mass $M$ and accretion rate $\dot{M}$. We average the accretion rates over 300 years, to take into account the transport of material through the unresolved parts of the accretion disks, as in H16. In addition, no strong time variation of $\dot{M}$ is observed in the late phase of our run when the radiative feedback is significant partly because protostars acquire mass through disk accretion driven by the gravitational torque of spiral arms rather than through mergers of clumps (see Stacy et al. 2016). We can therefore neglect the dependence of stellar properties on the accretion history (but see also H16 for the case the accretion history matters).

The RT of the direct photons from each protostar is solved with the adaptive ray-tracing (ART) method (Abel \& Wandelt 2002; Wise \& Abel 2011; Rosen et al. 2017; Kim et al. 2017), with which the rays are adaptively split to ensure the minimum number of rays per each cell, $N_{\text {ray }}=3$. Along each ray, we calculate the absorption of EUV photons by $\mathrm{H}$ ionization and the FUV photons by $\mathrm{H}_{2}$ self-shielding, as in $\mathrm{H} 16$.

Our computational domain is a cube with the side length $L_{\text {box }}=5 \times 10^{5}$ au. We set the base grids with $N_{\text {base }}=128$ cells in each direction and adaptively refine the cells to resolve one Jeans length with at least 16 cells. We set the maximum refinement level $l_{\max }=10$, resulting in the minimum cell size of $\Delta x_{\min }=L_{\text {box }} / N_{\text {base }} \times 2^{-l_{\max }} \approx 4$ au.

\subsection{Initial condition}

We simulate the formation of a Pop III star binary/multiple system in a fully cosmological context. To this end, we pick up a typical primordial star-forming cloud from more than 1600 samples obtained in the previous cosmological $3 \mathrm{D} \mathrm{SPH} / N$-body simulations (Hirano et al. 2014, 2015). The cloud we have chosen is the same as the case $\mathrm{C}$ of $\mathrm{H} 16$, for which they found the mass of the formed star has the median value among the five cases examined. This cloud begins to collapse at $z=24$ in a minihalo of $2 \times 10^{5} M_{\odot}$. We start our radiation hydrodynamics simulation by remapping the particle-based cosmological simulation data to our Cartesian grids when the central density reaches $10^{6} \mathrm{~cm}^{-3}$. We stop the simulation at $1.2 \times 10^{5} \mathrm{yr}$ after the first protostar formation, when the accretion is almost terminated and the final stellar masses are fixed. At the end, the simulation has required 9 months with 512 cores.

\section{RESULTS}

Fig. 1 shows the time evolution of the sink particles, i.e., protostars, appearing in our simulation. In the figure, masses, accretion rates, and separations are plotted from top to bottom. We give IDs S1-7 to the protostars in order of the formation time. According to qualitative transitions of the system, we define five evolutionary phases: (a) gravitational collapse, (b) initial fragmentation, (c) binary accretion, (d) late-time fragmentation, and (e) photo-evaporation. For each 


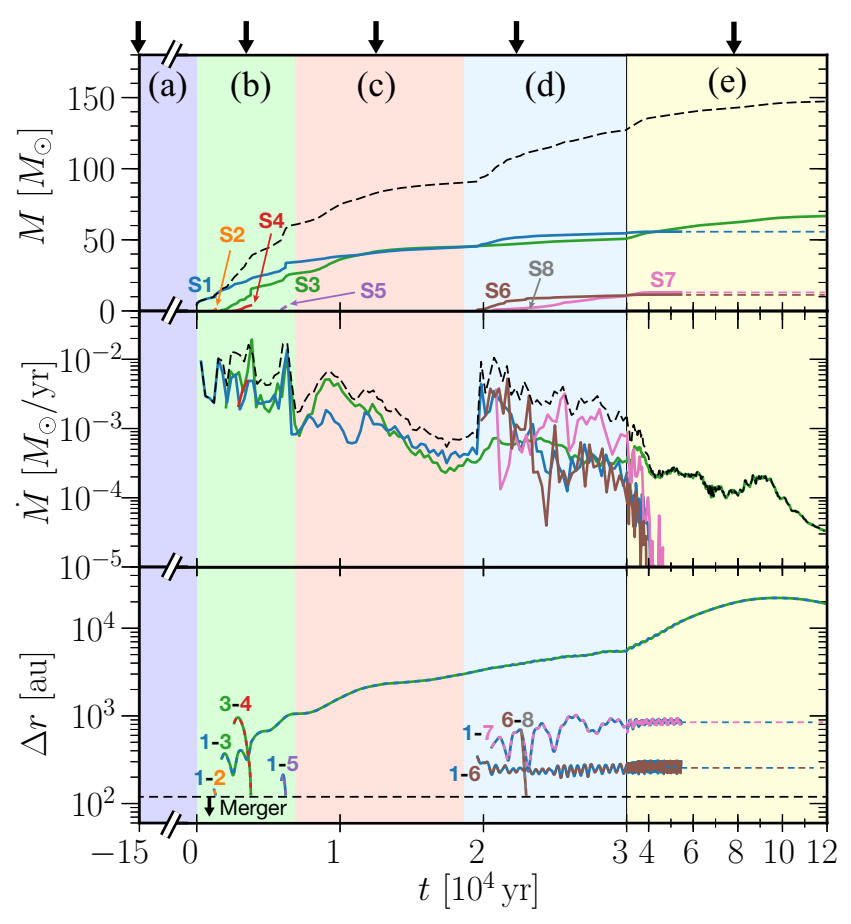

Figure 1. Time evolution of the mass (top), accretion rate (middle), and separation (bottom) of each protostar. The time $t$ is measured from the first protostar formation, which happens $1.5 \times 10^{5} \mathrm{yr}$ after the start of the simulation. The labels (a)-(e) shown at the top correspond to the evolutionary phases discussed in the text. The line colors represent the IDs of the protostars indicated in the top panel. In the top and middle panels, the dashed lines show the total mass and accretion rate, respectively. In the bottom panel, we plot the separations of the pairs of protostars whose IDs are indicated with the pairs of numbers, with the horizontal dashed line showing the threshold separation for merger. We do not solve the individual dynamics of S1, S6, and S7 after $t=5.5 \times 10^{4} \mathrm{yr}$, as described in the text. The top black arrows mark the times at which we present snapshots in Fig. 2.

evolutionary phase, we show a snapshot of the face-on surface density and edge-on temperature in Fig. 2. In the late-time fragmentation phase, we also present a 3D rendering view in Fig. 3. Below, we will describe how star formation proceeds in our simulation, tracing the five evolutionary stages.

(a) Gravitational collapse. We start our simulation from the initial condition of the cloud $1.5 \times 10^{5} \mathrm{yr}$ before the first protostar formation (Fig. 2a). The gravitational collapse proceeds in a self-similar fashion (Larson 1969; Penston 1969), with the central core having an oblate shape due to the rotation of gas.

(b) Initial fragmentation. As the maximum density increases as a result of the self-similar collapse, the first protostar, S1, forms at the center of the rotating core. Subsequently, the gas with finite angular momen-
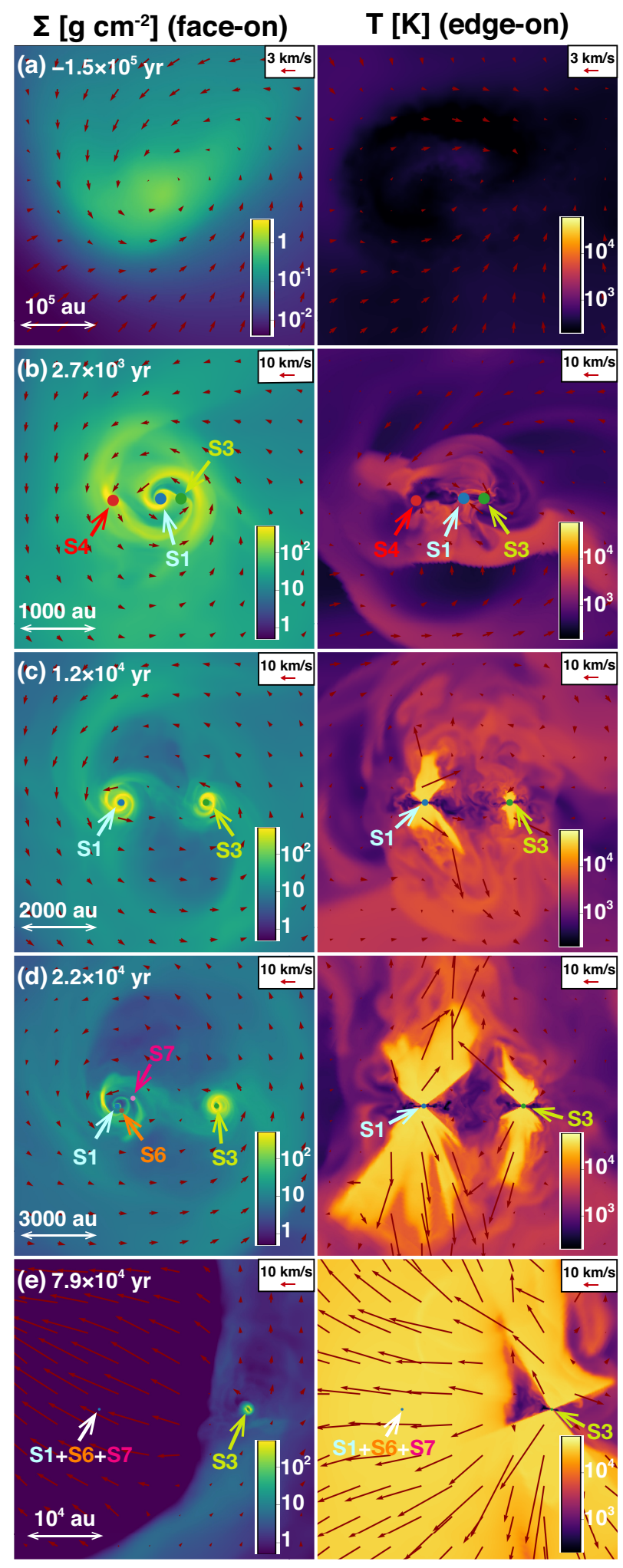

Figure 2. Snapshots of the gas distribution and protostar configuration in each of the five evolutionary phases. We show the face-on view of the surface density field with density-weighted velocity (left) and the edge-on sliced temperature field with velocity (right), along with the positions of protostars (thick arrows). The times of snapshots presented here are marked with the arrows in Fig. 1. 


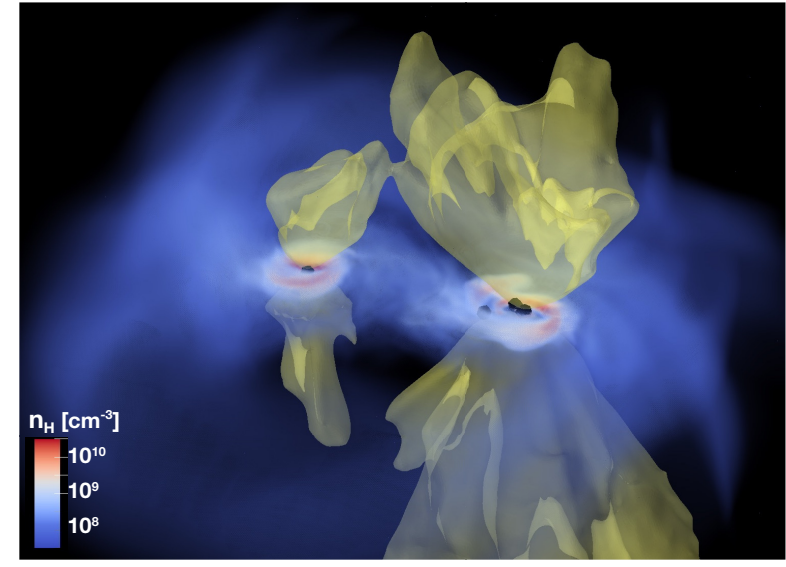

Figure 3. Volume rendering of the density field, together with ionization fronts (yellow surfaces) and protostars (black dots), $2.2 \times 10^{4} \mathrm{yr}$ after the first protostar formation (the same time as panel (d) of Fig. 2). Bipolar ionized bubbles are formed around both of the single-star (left) and minitriplet (right) systems.

tum falls to the vicinity of S1 and a circum-stellar disk is formed. The disk is highly gravitationally unstable because of its relatively high mass compared with the newly forming central protostar and vigorously fragments into several protostars (Fig. 2b). Most of them, however, do not survive as a result of accretion on the central star after inward migration through the disk or merger with others by three-body scattering.

(c) Binary accretion. After the initial fragmentation phase, only two stars, S1 and S3, survive and make up a binary system. They are surrounded by a circumbinary disk and each of them has its own circum-stellar disk (Fig. 2c). The gas accretes from the circum-binary disk to the circum-stellar disks and each star acquires the mass from its own circum-stellar disk. The accretion drives the binary evolution in mass and orbit: the total mass increases keeping the mass ratio around unity, and the separation increases due to accretion of higher angular momentum gas from the circum-binary disk. At this moment, double bipolar ionized bubbles with $T \gtrsim 3 \times 10^{4} \mathrm{~K}$ grow around the binary stars as their EUV emissivities rise (Fig. 2c, right). Note that this phenomenon could not be captured by previous simulations, which could treat only the central radiation source (e.g., Stacy et al. 2016; H16), and become tractable for the first time with our multi-source RT with the ART method. The accretion rate begins to decrease as the flows in the polar directions are quenched by the bubbles and the gas supply continues only in the equatorial directions.

(d) Late-time fragmentation. Due to imbalance between accretion rate from the circum-binary disk to the circum-stellar disks and that from circum-stellar disks to the stars, the gas accumulates on the circumstellar disks. As a result, the circum-stellar disk of S1 fragments into a few objects (Fig. 2d and 3). After a series of scatter and merger events, a mini-triplet system emerges, with newly formed companion stars (S6 and S7) orbiting around the massive central star (S1). Rapid accretion onto the companions embedded in the disk, together with the enhanced accretion onto the central object by non-axisymmetries, quickly exhausts the disk material. Due to the hierarchical structure of the mini-triplet system both in mass and distance, the orbits of the component stars remain nearly stable. The ionized bubbles continue expanding with more ionizing radiation being emitted as the stars become more massive and with the density in the surrounding envelope decreasing as the collapse of natal cloud proceeds.

(e) Photo-evaporation. Thereafter, the disk in the mini-triplet system, which has already depleted significantly by the accretion onto the stars, is lost by the photo-evaporation due to the radiation from the central massive star. As a consequence, the ionized bubble around the mini-triplet expands and merges with that around the star S3 (Fig. 2e). The accretion rate onto S3 is diminished gradually by the radiation not only by S3 itself but also by the mini-triplet. The distance between S3 and the mini-triplet becomes larger partly because the gravitational binding becomes weaker due to the loss of the gas in between them by the photoevaporation. At $5.5 \times 10^{4} \mathrm{yr}$ after the first protostar formation, we replace the mini-triplet system with a single sink particle that represents the gravity center, to save the computational cost. Since the properties of the mini-triplet system, namely, the masses and separations among member stars, hardly changed for the last $10^{4} \mathrm{yr}$, we assume that they remain unchanged in the rest of the simulation.

We stop our simulation at $1.2 \times 10^{5} \mathrm{yr}$ after the first protostar formation, when the mass of S3 almost reaches its final value with the accretion rate already reduced to $\dot{M} \sim 3 \times 10^{-5} M_{\odot} \mathrm{yr}^{-1}$. Extrapolating this decreasing trend of $\dot{M}$ in time, we find the mass of S3 increases by at most $\lesssim M_{\odot}$. We thus expect that further mass growth of S3 is insignificant. This means that the star formation process has been practically completed at the end of the simulation.

The end product at the final time step is a massive binary system consisting of stars with 56 and $67 M_{\odot}$ orbiting each other at a wide separation of $2 \times 10^{4} \mathrm{au}$. The $56 M_{\odot}$ star is also a member of a mini-triplet system with smaller mass companions with 12 and $13 M_{\odot}$ orbit- 
ing at 200 and $800 \mathrm{au}$, respectively. We have observed the formation of massive Pop III star binaries starting from the cosmological initial condition.

\section{DISCUSSION}

In this Letter, we have investigated the formation of a Pop III stellar system with an initial condition of a typical primordial star-forming cloud, by way of radiation hydrodynamics simulation. When the radiation feedback from the forming protostars quenches the gas supply, a binary system consisting of nearly equal-mass massive stars emerges, with one of the stars making up its own mini-triplet system with less-massive close companion stars. Although we have examined only one case in this Letter, our results suggest that Pop III stars are commonly formed as massive binary/multiple systems.

In spite of the same initial condition, we have observed the formation of multiple stars while only a single star was formed in the previous simulation of H16. This difference may come from a difference in resolution. In H16, only a single star survives possibly because of artificial mergers due to the insufficient resolution around companion stars. Besides, the total mass in stars $\left(\sim 150 M_{\odot}\right)$ in our simulation is smaller than that of the single star $\left(\sim 300 M_{\odot}\right)$ in H16, which may be related to the fact that the single star always resides at the center of the cloud, where the accretion is not easily quenched by the protostellar feedback because of high density. The total mass being shared with several stars, the mass of an individual object is even smaller in our simulation. This result should be taken into account in discussing the subsequent evolution of the Universe as the intergalactic-scale feedback from the Pop III stars, e.g., types of SNe (e.g., Woosley et al. 2002), largely depends on their masses.

The number of stars obtained in our simulation should be regarded as the lower limit because of our usage of the sink particle technique and ignorance of what may happen inside the sink particles, such as fragmentation. Moreover, although we assume that overlapping sink particles merge, two or more stars may continue orbiting each other at a distance shorter than the sink radius. Each sink particle, however, is likely to contain at least one massive star because a system consisting only of a large number of low-mass stars is unstable due to mutual gravitational scatterings. Therefore, our conclusion that Pop III stars typically form as massive binary/multiple systems is not altered by the possible existence of unresolved objects.

The binary system with 60 and $70 M_{\odot}$ stars found in our simulation is massive enough to be a progenitor of the binary $\mathrm{BH}$ mergers observed in gravitational waves (e.g., Kinugawa et al. 2014; Hartwig et al. 2016; Abbott et al. 2016; Belczynski et al. 2017) while the separation is too wide for any interaction to shrink the orbit in the late stage of stellar evolution and the remnants are unlikely to merge within the age of the Universe. To examine whether Pop III stars can be the progenitors of the observed BH binaries, the distribution of binary separations needs to be known. This is also crucial for predicting the abundance of Pop III X-ray binaries, which contribute to heating up the intergalactic medium in the epoch of reionization. For this purpose, we plan to perform similar simulations with a large number of samples in the future. Note that both the masses and separation of our binary system are located near the upper end of the statistical distributions of Pop III binaries given by Stacy \& Bromm (2013), who took their samples $5 \times 10^{3} \mathrm{yr}$ after the first protostar formation. The masses and separation of our system are already on the large side in the distributions by Stacy \& Bromm (2013) at the same timing and become even larger during the later evolution (see Fig. 1).

Aside from the massive wide binary, we have also seen the formation of the mini-triplet system. Its separation is much smaller than that of the massive binary because it is formed from the circum-stellar disk, which has lower angular momentum compared with the original cloud. On the contrary, the massive binary has a large separation as a result of the accretion of high angular momentum gas from the cloud. In fact, we have seen that the separation of the binary increases as it accretes the gas from the circum-binary disk, as suggested in recent simulations of binary accretion (Muñoz et al. 2019; Moody et al. 2019; Duffell et al. 2019). The late-time disk fragmentation leading to such multiple systems may play some roles in formation of close binaries, which can evolve to the progenitors of $\mathrm{BH}$ merger events or X-ray binaries. Additionally, other mechanisms, such as a-fewbody scatterings of protostars and angular momentum extraction by magnetic fields, if any, might help shape the close binaries.

We have succeeded in seeing a new evolutionary aspect of the Pop III star formation. This is, however, just the beginning of the attempts toward its thorough understandings as some more processes, such as subsink scale physics, are still to be clarified. Those issues need to be addressed in future studies.

The authors thank Sunmyon Chon, Michiko Fujii, Masahiro Machida, Massimo Ricotti, Hajime Susa, Hidekazu Tanaka, Ataru Tanikawa, Masauyuki Umemura, Hidenobu Yajima, and Naoki Yoshida for fruitful discussions and comments. K.S. and S.H. 
appreciate the support by the JSPS Overseas Research Fellowship and JSPS Research Fellowship, respectively. This work is supported in part by MEXT/JSPS KAKENHI Grant Number 17H02863, 17K05394, 18H05436, 18H05437 (T.M.), 16H05996, $19 \mathrm{H} 09134$ (T.H.), 18J01296 (S.H.), and 17H01102,
17H02869, 17H06360 (K.O.). The numerical simulations were performed on the Cray XC50 at CfCA of the National Astronomical Observatory of Japan, the computer cluster Draco at Frontier Research Institute for Interdisciplinary Sciences of Tohoku University, and the Cray XC40 at Yukawa Institute for Theoretical Physics in Kyoto University.

\section{REFERENCES}

Abbott, B. P., Abbott, R., Abbott, T. D., et al. 2016, Physical Review Letters, 116, 061102

Abel, T., Bryan, G. L., \& Norman, M. L. 2002, Science, 295, 93

Abel, T., \& Wandelt, B. D. 2002, MNRAS, 330, L53

Belczynski, K., Ryu, T., Perna, R., et al. 2017, MNRAS, 471, 4702

Bromm, V., Coppi, P. S., \& Larson, R. B. 2002, ApJ, 564, 23

Chon, S., \& Hosokawa, T. 2019, MNRAS, 488, 2658

Clark, P. C., Glover, S. C. O., Smith, R. J., et al. 2011, Science, 331, 1040

Dewdney, P. E., Hall, P. J., Schilizzi, R. T., \& Lazio,

T. J. L. W. 2009, IEEE Proceedings, 97, 1482

Duffell, P. C., D’Orazio, D., Derdzinski, A., et al. 2019, arXiv e-prints, arXiv:1911.05506

Glover, S. 2013, Astrophysics and Space Science Library, Vol. 396, The First Stars, ed. T. Wiklind, B. Mobasher, \& V. Bromm, 103

Greif, T. H. 2015, Computational Astrophysics and Cosmology, 2, 3

Greif, T. H., Bromm, V., Clark, P. C., et al. 2012, MNRAS, 424, 399

Greif, T. H., Springel, V., White, S. D. M., et al. 2011, ApJ, 737, 75

Hartwig, T., Volonteri, M., Bromm, V., et al. 2016, MNRAS, 460, L74

Hirano, S., \& Bromm, V. 2017, MNRAS, 470, 898

Hirano, S., Hosokawa, T., Yoshida, N., Omukai, K., \& Yorke, H. W. 2015, MNRAS, 448, 568

Hirano, S., Hosokawa, T., Yoshida, N., et al. 2014, ApJ, 781,60

Hosokawa, T., Hirano, S., Kuiper, R., et al. 2016, ApJ, 824, 119

Hosokawa, T., \& Omukai, K. 2009, ApJ, 691, 823

Hosokawa, T., Omukai, K., Yoshida, N., \& Yorke, H. W. 2011, Science, 334, 1250

Hosokawa, T., Yorke, H. W., \& Omukai, K. 2010, ApJ, 721, 478
Jeon, M., Pawlik, A. H., Bromm, V., \& Milosavljević, M. 2014, MNRAS, 440, 3778

Kim, J.-G., Kim, W.-T., Ostriker, E. C., \& Skinner, M. A. 2017, ApJ, 851, 93

Kinugawa, T., Inayoshi, K., Hotokezaka, K., Nakauchi, D., \& Nakamura, T. 2014, MNRAS, 442, 2963

Larson, R. B. 1969, MNRAS, 145, 271

Matsumoto, T. 2007, PASJ, 59, 905

Matsumoto, T., Dobashi, K., \& Shimoikura, T. 2015, ApJ, 801,77

McKee, C. F., \& Tan, J. C. 2008, ApJ, 681, 771

Mirabel, I. F., Dijkstra, M., Laurent, P., Loeb, A., \& Pritchard, J. R. 2011, A\&A, 528, A149

Moody, M. S. L., Shi, J.-M., \& Stone, J. M. 2019, ApJ, 875,66

Muñoz, D. J., Miranda, R., \& Lai, D. 2019, ApJ, 871, 84

Omukai, K., \& Inutsuka, S.-i. 2002, MNRAS, 332, 59

Omukai, K., \& Nishi, R. 1998, ApJ, 508, 141

Omukai, K., \& Palla, F. 2003, ApJ, 589, 677

Penston, M. V. 1969, MNRAS, 144, 425

Rosen, A. L., Krumholz, M. R., Oishi, J. S., Lee, A. T., \& Klein, R. I. 2017, Journal of Computational Physics, 330, 924

Sharda, P., Krumholz, M. R., \& Federrath, C. 2019, MNRAS, 490, 513

Smith, R. J., Glover, S. C. O., Clark, P. C., Greif, T., \& Klessen, R. S. 2011, MNRAS, 414, 3633

Stacy, A., \& Bromm, V. 2013, MNRAS, 433, 1094

Stacy, A., Bromm, V., \& Lee, A. T. 2016, MNRAS, 462, 1307

Stacy, A., Greif, T. H., \& Bromm, V. 2010, MNRAS, 403, 45

-. 2012, MNRAS, 422, 290

Susa, H. 2019, ApJ, 877, 99

Susa, H., Hasegawa, K., \& Tominaga, N. 2014, ApJ, 792, 32

Tan, J. C., \& McKee, C. F. 2004, ApJ, 603, 383

Wise, J. H., \& Abel, T. 2011, MNRAS, 414, 3458

Woosley, S. E., Heger, A., \& Weaver, T. A. 2002, Reviews of Modern Physics, 74, 1015

Yoshida, N., Omukai, K., \& Hernquist, L. 2008, Science, 321,669 